\title{
Nanoscale Properties of Human Telomeres Measured with a Dual Purpose X-ray Fluorescence and Super Resolution Microscopy Gold Nanoparticle Probe
}

J. Charles G. Jeynes, ${ }^{* \dagger \odot}$ Kalotina Geraki, ${ }^{\ddagger}$ Christopher Jeynes, ${ }^{\S}$ Mi Zhaohong, ${ }^{\|}$Andrew A. Bettiol, ${ }^{\| \odot ~}$ Eva Latorre, ${ }^{\perp}$ Lorna Wendy Harries, ${ }^{\perp}$ and Christian Soeller ${ }^{\#}$

${ }^{\dagger}$ Centre for Biomedical Modelling and Analysis, University of Exeter, Exeter, Devon, U.K., EX2 5DW

${ }^{\ddagger}$ Diamond Light Source, Didcot, Oxfordshire U.K., OX11 0DE

${ }^{\S}$ Ion Beam Centre, University of Surrey, Guildford, Surrey, U.K., GU2 7XH

"Centre for Ion Beam Applications, National University of Singapore, Singapore, 119077

${ }^{\perp}$ Medical School, RILD Building, University of Exeter, Barrack Road, Exeter, Devon, EX2 5DW

${ }^{\#}$ Living Systems Institute \& Biomedical Physics, University of Exeter, Exeter, Devon, U.K., EX2 5DW

\section{Supporting Information}

ABSTRACT: Techniques to analyze human telomeres are imperative in studying the molecular mechanism of aging and related diseases. Two important aspects of telomeres are their length in DNA base pairs (bps) and their biophysical nanometer dimensions. However, there are currently no techniques that can simultaneously measure these quantities in individual cell nuclei. Here, we develop and evaluate a telomere "dual" gold nanoparticle-fluorescent probe simultaneously compatible with both X-ray fluorescence (XRF) and super resolution microscopy. We used silver enhancement to independently visualize the spatial locations of gold nanoparticles inside the nuclei, comparing to a standard QFISH (quantitative fluorescence in situ hybridization) probe, and showed good specificity at $\sim 90 \%$. For sensitivity, we calculated telomere length based on a DNA/gold binding ratio using XRF and compared to quantitative polymerase chain reaction (qPCR) measurements. The
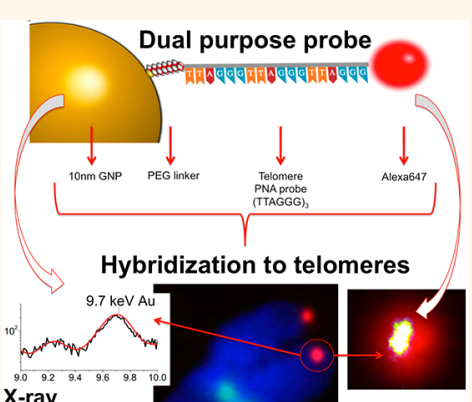
X-ray fluorescence sensitivity was low $(\sim 10 \%)$, probably because of steric interference prohibiting the relatively large $10 \mathrm{~nm}$ gold nanoparticles access to DNA space. We then measured the biophysical characteristics of individual telomeres using super resolution microscopy. Telomeres that have an average length of $\sim 10 \mathrm{kbps}$, have diameters ranging between $\sim 60-300 \mathrm{~nm}$. Further, we treated cells with a telomere-shortening drug and showed there was a small but significant difference in telomere diameter in drug-treated vs control cells. We discuss our results in relation to the current debate surrounding telomere compaction.

KEYWORDS: telomeres, super resolution microscopy, X-ray fluorescence, nanoparticles, dSTORM

$\mathrm{T}$ elomeres are repetitive sequences located at the ends of chromosomes. They have a number of functions including protecting the chromosomes from degradation and preventing individual chromosomes from linking to each other. They are also associated with the aging process and shorten with each cell replication cycle. ${ }^{1}$ It is accepted that at least $8-10$ base pairs (bps) are lost per cell division due to the "end replication problem", 2,3 while many more bps are thought to be eroded due to reactive oxygen species. Measuring these dynamics is important but technically challenging.

Further, the packing density of the DNA in telomeres is currently a focus of research. Bandaria et al. ${ }^{4}$ recently proposed that DNA damage response (DDR) complexes are unable to enter into telomeric regions due to DNA compaction. However, Timashev et al. ${ }^{5}$ and Vancevska et al. ${ }^{6}$ questioned this hypothesis as they found DDR complexes could colocalize with telomeres, even when purported compaction proteins were present. Thus, measuring biophysical dimensions of telomeres is important, as volume and packing density could play a vital role in biological function.

Received: October 5, 2017

Accepted: November 1, 2017

Published: November 1, 2017 

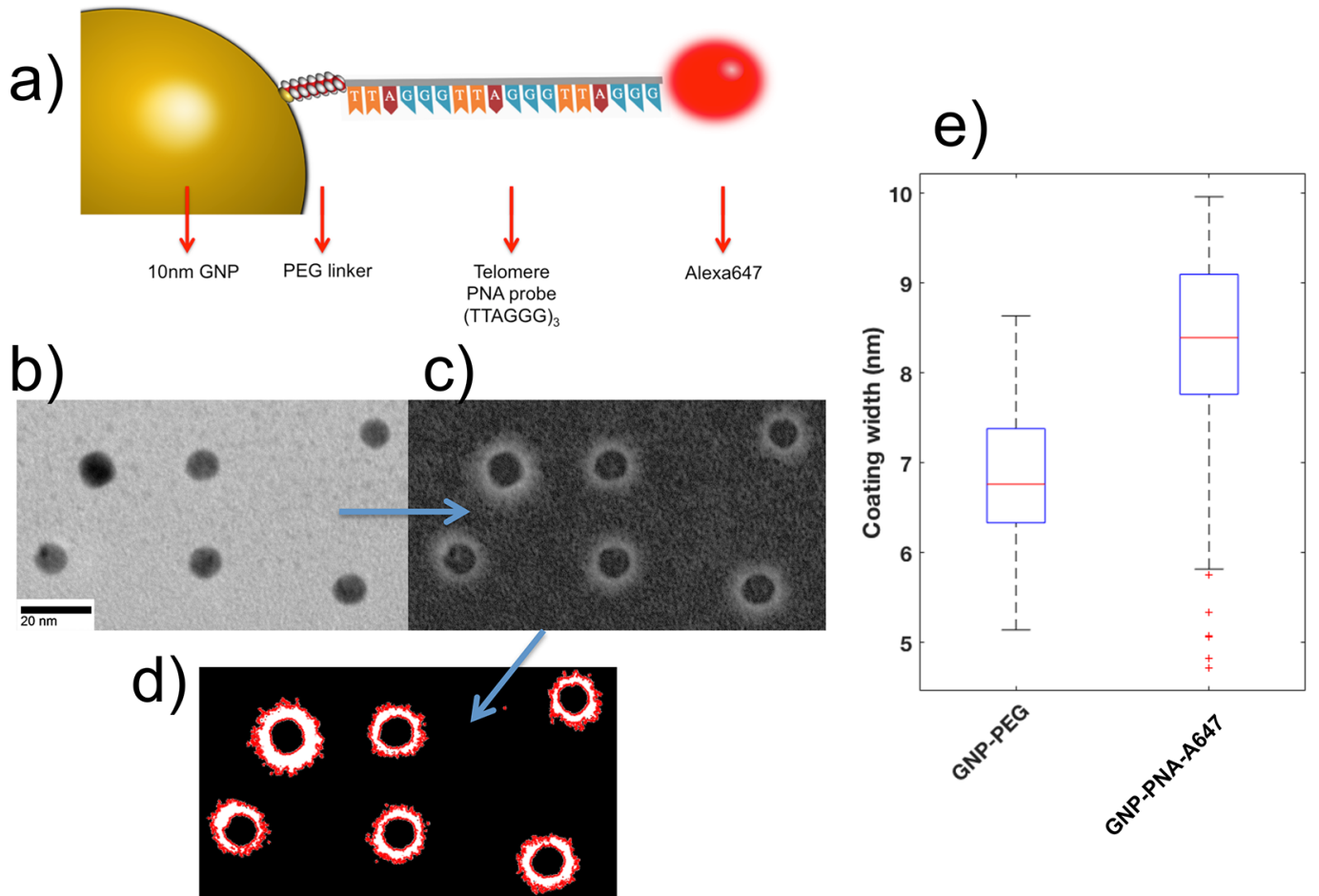

Figure 1. (a) Schematic of the probe design, GNP-PNA-A647. A $10 \mathrm{~nm}$ gold nanoparticle is covalently linked to a peptide nucleic acid (PNA) probe through a PEG-linker molecule. The PNA probe has an Alexa-647 fluorescent molecule at the N-terminus. At either end of the PNA probe there is also a spacer molecule (for details see the Supporting Information); Lys-O-CCCTAACCCTAACCCTAA-O-Lys(Alexa-647). (b) Unprocessed representative transmission electron microscope image of GNP-PNA-A647 conjugates. (c) Image is background subtracted (see the Supporting Information) to enhance the contrast of the organic shell. (d) Image is then automatically segmented using an Otsu threshold and the shell properties (e.g., area) are measured. (e) Analysis of the shell coating between GNP-PEG $(N=51)$ and GNP-PNA-A647 (N=59) shows a slight increase in the width, with medians significant $(P=0.001)$ using a Mann-Whitney-U test. The boxes have lines at the lower quartile, median and upper quartile values. The whiskers show the extent of the rest of the data, and the crosses are outliers.

There are a number of ways to measure telomere length (in base pairs of DNA), while there are very few methods that can measure biophysical characteristics, like volume. Methods such as southern hybridization can measure average telomere length in bulk homogenate samples. ${ }^{7}$ Bulk methods mask the heterogeneity that could occur at each telomere within a nucleus. ${ }^{8,9}$ For individual telomeres, a method known as quantitative fluorescence in situ hybridization (QFISH) uses a short fluorescence oligonucleotide sequence (made of DNA, or more commonly, PNA) probe for the repeat motif found in telomeres, visualized with standard fluorescence microscopy. ${ }^{10}$ Preparing the cells so that they are in the metaphase allows telomeres to be seen directly on the ends of each chromosome. ${ }^{11}$ The length of each telomere is usually estimated based on fluorescence intensity in arbitrary fluorescence units, or as a ratio to a centromeric probe of known length.

However, conventional QFISH measurements do not directly measure the absolute length of the DNA nor do they measure the actual spatial dimensions of the telomere due to the light diffraction limitations of standard fluorescence imaging. Here we attempt to measure both of these quantities using a probe that is compatible with both X-ray fluorescence (XRF) and super resolution microscopy (here using dSTORM; direct stochastic reconstruction optical microscopy $\left.{ }^{12}\right)$. We chose XRF as it has excellent sensitivity to gold and can be absolutely quantitative in measuring numbers of atoms in a sample as well as having micron resolution.
We conducted a correlative approach where the two measurements are obtained on identically prepared samples and found that our probe could be used with both imaging modalities. We discuss the limitations and potential improvements that could be made to the method. We also compare our super resolution results to limited literature values and discuss the sensitivity of the technique as a method of determining telomere diameter (or volume).

\section{RESULTS}

We constructed the probe using a gold nanoparticle conjugation kit (Creative-Diagnostics, NY) and a custommade fluorescent peptide nucleic acid (PNA) oligonucleotide (Panagene, Korea). The two components were conjugated together using the manufacturers protocol (for details, see Experimental Methods and the Supporting Information). We call our probe GNP-PNA-A647 to represent the important components (for schema, see Figure 1a).

The gold nanoparticles are ideal for detection through the characteristic XRF signal for the Au L-shell, while Alexa-647 is an organic dye that is widely used in super resolution microscopy. By quantifying the GNP signal, it is possible to infer how many DNA bases there are in a telomere, if we can obtain an estimate of the ratio of GNPs to DNA bases. Quantifying the A647 signal using super resolution microscopy provides a measure of the actual spatial dimension of the telomere, rather than the much larger apparent area that is commonly obtained using diffraction-limited modalities, such as confocal microscopy. 

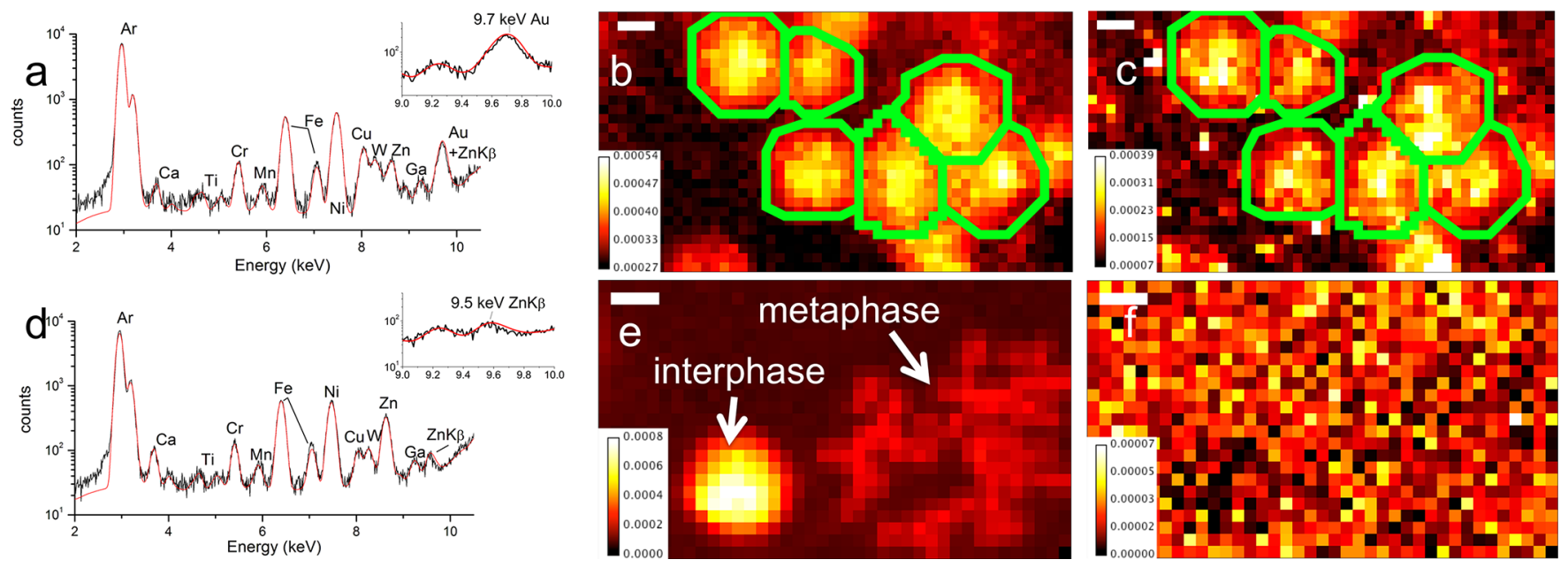

Figure 2. Sensitivity of the probe (a) X-ray fluorescence (XRF) spectrum from a $60 \mu \mathrm{m} \times 100 \mu \mathrm{m}$ scanned region, containing 6 cell nucleus probed with GNP-PNA-A647. The counts (black line) are fitted (red line) so that each peak can be assigned to an element and quantified. The gold peak at $9.7 \mathrm{keV}$ is magnified in the inset. (b) Elemental $\mathrm{Zn}$ map made by displaying just the $\mathrm{Zn}$ peak from the spectrum shown in part a. The pixel intensity scale bar shows the mass fraction of $\mathrm{Zn}$. Each cell nucleus is segmented (green outline), and a mass fraction average is measured for each nucleus. The white scale bar is $10 \mu \mathrm{m}$. (c) Au map of the same region. The average Au mass fraction per nucleus is shown in Table 1, converted to numbers of gold nanoparticles. (d) XRF spectrum from control cells. (e) Zn map from control cells. Note the faint lines are chromosomes from a metaphase spread (f) Au map from control cells. There is no Au signal distinguishable above the background noise. The GNP-PNA-A647 probe measured telomere length on average to $\sim 0.9 \mathrm{kbps}$ (see Table 1 and Table SI 1). By comparing this length to the qPCR length measurement (see Figure 5), our probe has a sensitivity of $\sim 10 \%$.

We characterized the GNP-PNA-A647 probe, primarily using transmission electron microscopy (TEM) and automated image analysis (see Figure $1 \mathrm{~b}-\mathrm{e}$, and Experimental Methods for image analysis techniques). Figure le shows the equivalent diameters of the organic shell surrounding GNPs, comparing GNPs (direct from the manufacturer) and GNP-PNA-A647. Note the GNPs from the manufacturer are functionalized with a $5 \mathrm{kDa}$ polyethylene glycol (PEG) layer, thus we call them GNP-PEG. The median shell diameter was $\sim 6.5 \mathrm{~nm}$ and $\sim 8.5$ $\mathrm{nm}$ for GNP-PEG vs GNP-PNA-647, respectively, a statistically significant difference using a Mann-Whitney-U-test.

Our GNP-PEG measurement is largely in agreement with del Pino et al., ${ }^{13}$ who measured a $\sim 7-10 \mathrm{~nm}$ diameter shell on their GNP-PEG constructs, also using a $5 \mathrm{kDa}$ PEG molecule. In addition to TEM measurements, we used UV-vis spectroscopy to measure the characteristic Alexa647 absorption peak at $\sim 647 \mathrm{~nm}$, which is present in our purified probe construct but not present in GNP-PEG samples (see SI Figure 1).

Next, we hybridized our GNP-PNA-A647 probe to telomeres on human cells. We used a standard metaphase spread protocol using Human Embryonic Kidney (HEK-293) cells (see Experimental Methods) to produce well spread out chromosomes on which to perform probe hybridization. We used an in situ fluorescence hybridization (FISH) kit (Dako, Denmark), replacing their probe with our own. It should be noted our FISH protocol (and most FISH protocols in general) uses proteases and ribonucleases to strip the cell of all components other than the DNA contained in the nucleus. Thus, the images shown are solely from DNA. As a control, we probed the cells with GNPs that lack the PNA-A647.

HEK cells are known to tolerate colcemid that blocks replication at the metaphase, which facilitated reproducibly harvesting a large number of interphase nuclei and, to a lesser extent, metaphase chromosomes. These can be distinguished based on their appearance: interphase nuclei are circular as all the individual chromosomes are clustered together in a circular arrangement; by contrast, the chromosomes in metaphase have condensed and separated so that that they can be seen individually.

There were technical reasons why we did not concentrate on metaphase spreads with XRF: beam targeting and time constraints on the XRF system required focusing on the more numerous interphase nuclei since metaphase spreads are much less common than interphase nuclei in a sample preparation.

Probe Sensitivity. The XRF imaging of the samples was performed with the microprobe of the Microfocus Spectroscopy beamline I18 at the Diamond Light Source. ${ }^{14}$ The data collection time on each pixel was $10 \mathrm{~s}$, which meant that each region scan generally took about $4 \mathrm{~h}$, depending on the size of the scan. The beam spot size was $2.5 \mu \mathrm{m}$ giving the pixel size in the images in Figure 2. Figure $2 a-c$ shows an example of a region of six nuclei probed with GNPs-PNA-A647. The spectrum (Figure $2 \mathrm{a}$ ) shows a number of elements associated with cells (e.g., $\mathrm{Zn}, \mathrm{Fe}, \mathrm{Cu}$ ) while others are background from the sample stage (e.g., $\mathrm{Ga}, \mathrm{Ti}$ ) or air (Ar).

Processing of the raw data was done with PyMCA software and involves peak fitting, background removal, and estimation of concentration levels with the use of a reference material (AXO, Dresden $\mathrm{GmbH}$ ). In PyMCA, the reference material is modeled in terms of matrix composition, density, and thickness and is used to determine the photon flux of the experiment. The photon flux value is used in conjunction with the matrix, density, and thickness of our probed samples to measure the concentration of gold. The matrix and density assumed for our samples was the International Commission on Radiation Protection (ICRP) standard soft tissue composition (CNHO, $\left.\mathrm{g} / \mathrm{cm}^{3}\right)$. We measured the thickness of the cell nuclei using two independent techniques. We used ion beam analysis (IBA) ${ }^{15}$ and measured an average nucleus thickness of $\sim 45 \mathrm{~nm}$ (for details please see the Supporting Information). For comparison, 
Table 1. Quantification of the Au Signal from Each of the Six Cell Nuclei Shown in Figure 1a-c, from Left to Right ${ }^{a}$

\begin{tabular}{|c|c|c|c|c|c|c|}
\hline $\begin{array}{c}\text { cell } \\
\text { number }\end{array}$ & $\begin{array}{l}\text { mass of the cell nucleus } \\
(\mathrm{pg})\end{array}$ & $\begin{array}{l}\text { std dev of cell nucleus } \\
\text { mass }(\mathrm{pg})\end{array}$ & $\begin{array}{l}\text { mass of Au per cell } \\
\text { nucleus }(\mathrm{pg})\end{array}$ & $\begin{array}{c}\text { std dev of Au mass } \\
(\mathrm{pg})\end{array}$ & $\begin{array}{c}\text { gold nanoparticles } \\
\text { per cell }\end{array}$ & $\begin{array}{l}\text { bases of DNA per } \\
\text { telomere }\end{array}$ \\
\hline 1 & 34.0 & 8.8 & 0.457 & 0.084 & 3923 & 821 \\
\hline 2 & 20.3 & 5.2 & 0.258 & 0.048 & 2218 & 464 \\
\hline 3 & 32.0 & 8.2 & 0.391 & 0.072 & 3358 & 703 \\
\hline 4 & 33.3 & 8.6 & 0.460 & 0.085 & 3949 & 826 \\
\hline 5 & 33.7 & 8.7 & 0.492 & 0.091 & 4227 & 884 \\
\hline 6 & 31.3 & 8.0 & 0.406 & 0.075 & 3491 & 730 \\
\hline Mean & 30.8 & 7.9 & 0.411 & 0.076 & 3528 & 738 \\
\hline std dev & 5.2 & 1.4 & 0.083 & 0.015 & 716 & 150 \\
\hline
\end{tabular}

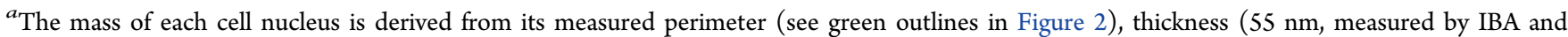
STIM) and density $\left(1 \mathrm{~g} / \mathrm{cm}^{3}\right)$. The standard deviation is calculated from the uncertainty of the cell nucleus thickness ( \pm 10 nm). The mass of the Au in each nucleus is calculated from the parts per million concentration measured by XRF. The gold nanoparticles are 10 nm in diameter, so assuming that each GNP binds to 18 bases of DNA and the each nucleus has 86 telomeres, the average number of bases per telomere can be calculated (see text for details)

we used scanning transmission ion microscopy $(\text { STIM })^{16}$ and measured an average thickness of $\sim 65 \mathrm{~nm}$ (see the Supporting Information for details) per nucleus. The two estimates are in reasonable agreement given the variability in sample preparation and the intrinsic sample heterogeneity. For XRF data fitting, we performed two fits assuming either a 45 or $65 \mathrm{~nm}$ nucleus thickness. The values for the gold concentration shown in Table 1 are the mean value of these two fits with standard deviation.

Figure $2 \mathrm{~b}, \mathrm{c}$ shows $\mathrm{Zn}$ and $\mathrm{Au}$ elemental concentration maps. The Au $\mathrm{L} \alpha$ peak at $9.7 \mathrm{keV}$ is clearly present in the GNP-PNAA647 probed cells. A similar XRF spectrum can be seen for the control (Figure 2d), where cell nuclei were probed with GNPs but without the PNA-A647 oligonucleotide. Here though, the $\mathrm{Zn}$ map reveals the cell nuclei (Figure 2e) but the Au map (Figure 2f) shows no discernible features.

We segmented the cells (areas shown in green) and extracted an average Au mass fraction per nucleus (i.e., the average pixel intensity). The mass fraction is multiplied by $10^{6}$ to give the ppm (parts-per-million by weight) concentration. The ppm concentration of gold can only be converted into absolute values if one knows the mass of the matrix, which in this case is the mass of the nucleus. The mass of the nucleus is derived from the measured perimeter and average nuclear thickness (55 $\mathrm{nm}$ ), given the ICRP soft tissue composition standard density $\left(\mathrm{g} / \mathrm{cm}^{3}\right)$.

Table 1 is a summary of measurements that allows estimates of the number of gold nanoparticles in each nucleus. This is calculated from the mass of gold in each nucleus, divided by the mass of a single gold nanoparticle (which is $1.01 \times 10^{-17} \mathrm{~g}$; the volume of a $10 \mathrm{~nm}$ sphere is $5.24 \times 10^{-19} \mathrm{cc}$ and the density of gold is $19.3 \mathrm{~g} / \mathrm{cm}^{3}$ ).

To arrive at an approximate number of gold nanoparticles per telomere region, we assumed a binding ratio of 1:18 GNP/ telomeric-DNA as determined by the design of our probe (see Figure 1), and we measured an average $86 \pm 23$ telomeres per nucleus by counting the number of Alexa- 647 fluorescent dots in $N=20$ interphase nuclei (see Figure $3 \mathrm{a}$ as an example: the variation in telomeres is probably due to aneuploidy in HEK cells, thus no two cells are likely to have exactly the same number of chromosomes and consequently telomeres). Finally, to calculate the number of DNA base pairs per telomere, the number of GNPs per nucleus is divided by 86 (to obtain GNPs per telomere) and then multiplied by 18 (binding ratio). For the 6 cells we measured shown in Figure 1, we obtain on average about 3500 GNPs per cell that equates to 740 DNA
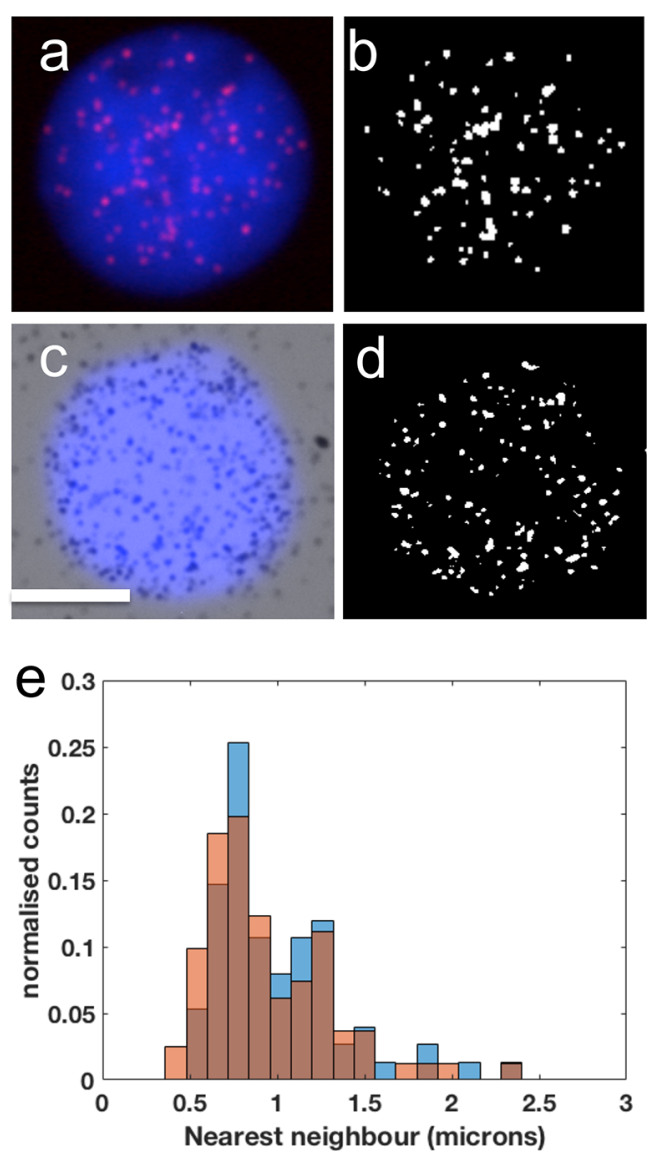

Figure 3. Specificity of the probe. X-ray fluorescence does not have the resolution for individual telomeres, so the specificity of the probe was measured using silver enhancement of the gold, compared to a conventional PNA probe (a) fluorescence signal from a conventional PNA probe, shown in an interphase nucleus. Part $b$ shows the automatic identification of the telomeres from part a using an Otsu threshold. Part $c$ shows brightfield light microscopy of a different nucleus, silver enhanced to show the locations of gold nanoparticles. The scale bar is $10 \mu \mathrm{m}$. Part $\mathrm{d}$ shows the automatic identification of the enhanced gold nanoparticles from part c. (e) The spatial distributions of the identified fluorescence regions and silver enhanced gold nanoparticles regions were compared with nearest neighbor analysis and are very similar, indicating good specificity. 
bases per telomere (see Table 1). When we combine this with data measured on other cells (see SI Figure 4), the average is $\sim 4400$ GNPs per cell, corresponding to $\sim 900 \mathrm{bps}$.

To measure the actual length of the average telomere in the HEK cells, we used quantitative polymerase chain reaction (qPCR), an established method for measuring telomere length. ${ }^{17}$ Briefly, a $84 \mathrm{mer}$ "telomere" PCR template (TGAC$\mathrm{CA})_{84}$ is used in a serial dilution to create a standard curve of telomere template concentration (equivalent to telomere length) against cycle time completion. Thus, unknown samples can be measured against this standard. DNA was extracted from the HEK cells and had on average $10.2 \pm 0.12 \mathrm{kbps}$ long telomeres (see Figure 4a). By comparing this length to the XRF

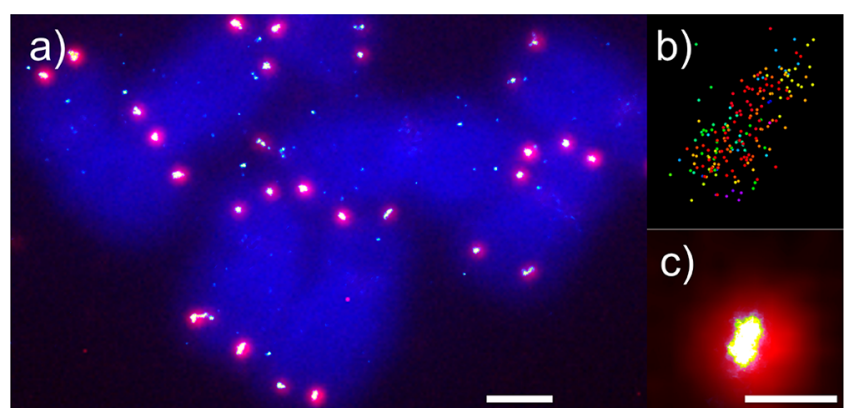

d)

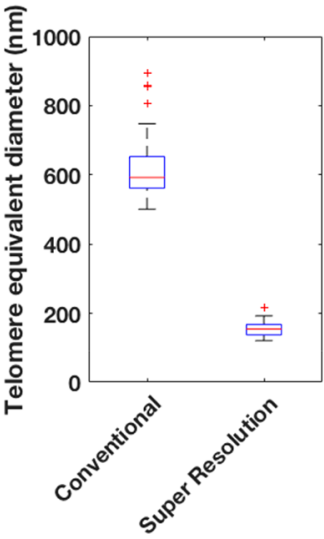

e)

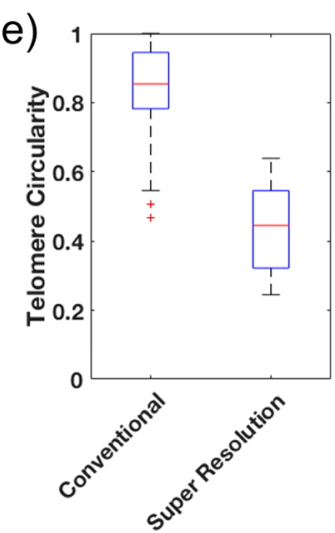

Figure 4. Analysis of telomeres dimensions comparing standard fluorescence with super resolution microscopy. (a) An example of a metaphase spread showing chromosomes (blue) with telomeres (red) taken using conventional diffraction limited fluorescence microscopy, overlaid with the super resolution image of the telomeres (white). The scale bar is $2 \mu \mathrm{m}$. (b) Example of the raw data from a super resolution image of a telomere, where each dot represents an event. (c) Magnified image of a telomere comparing conventional (red) and super resolution (white). The scale bar is $500 \mathrm{~nm}$. (d) Compares the equivalent diameters of telomeres from conventional vs super resolution microscopy (equivalent diameter $=\sqrt{ }(4 \times$ area $/ \pi))$. (e) Compares the circularity of telomeres from conventional vs super resolution microscopy (where $1=$ perfect circle and $0=$ ellipse).

length measurement using our probe (which gave an average telomere length of $0.9 \mathrm{kbps}$ ), our probe has a sensitivity of $\sim 10 \%$. Clearly, the GNPs are not fully covering the telomeric region, which could potentially be because of their relatively large size (see Discussion).

Probe Specificity. The X-ray beam size was far too large to directly visualize individual clusters of GNPs on individual telomeres. To confirm that GNP-PNA-A647 was binding specifically to the telomere regions rather than nonspecifically binding to random DNA, we used silver enhancement. This is a commonly used technique, where silver ions nucleate around GNPs and grow over time, eventually becoming large enough to be seen with light microscopy. For specificity analysis, we compared silver enhanced images (Figure 3c) to images hybridized with PNA-A647 (Figure 3a), a conventional probe. To quantify the images, we automatically segmented and counted the dots using a binary threshold (Figure 3b,d) and then took the ratio between the standard and our probe, as a measure of specificity. From $N>500$ dots, the specificity is 87.2\% (average 86 dots per PNA-A647 nucleus and an average of 75 dots per silver stained nucleus). As an additional measure, we also compared the spatial distribution of the dots between the standard and our probe, using nearest neighbor analysis. Figure $3 \mathrm{e}$ shows a histogram of the data. Here, the distributions are similar and the means are not significantly different in a Mann-Whitney-U test.

Super Resolution Analysis of Telomeres. As the next part of our investigation, we used super resolution microscopy to measure the biophysical dimensions of telomeres. Here, we utilized the "blinking" characteristics ${ }^{18}$ of the Alexa-647 molecule on our probe, visualized with direct stochastic reconstruction microscopy (dSTORM). The samples were mounted in an oxygen-scavenging buffer (for details see Experimental Methods). Probed nuclei were first imaged using diffraction limited fluorescence microscopy and then subsequently imaged using dSTORM for comparison.

Figure 4a shows a representative image of a number of chromosomes (blue), with the telomeres from light diffraction images (red) overlaid with the corresponding super resolution images (white). Figure $4 \mathrm{~b}$ shows the raw data from a super resolution acquisition from a single telomere and illustrates the $>200$ events from the region. This raw data is rendered into a super resolution image using a "jittered triangulation" algorithm, ${ }^{18}$ illustrated in a magnified view of a telomere in Figure 4c. An analysis of the spatial properties of telomeres was then conducted, in both diffraction limited and super resolution images, and the results are shown in Figure 4 d,e. Figure $4 d$ shows the equivalent diameters of the diffraction limited telomeres ranging between $\sim 550$ and $750 \mathrm{~nm}$, while the super resolution resolved telomeres range between 60 and $300 \mathrm{~nm}$. Figure $3 \mathrm{e}$ shows that the diffraction-limited telomeres are almost circular, while the super resolution resolved telomeres are ellipsoidal (or irregular). This comparison is important, as it shows how diffraction limited images can lead to erroneous conclusions. Previously it was thought that telomeres were spheroids, ${ }^{9}$ while our data shows they are, in general, ovoid or irregular. Furthermore, by measuring the actual dimensions of telomeres, as well as their length, the compaction density of the DNA can be estimated (see Discussion). The circularity (determining surface area) and the DNA density are both important when considering how enzymes enter into and interact with the telomere.

Figure 5 shows a comparison between HEK cells treated with the drug azidothymidine (AZT) and untreated control HEK cells. AZT is a putative telomerase inhibitor and has been shown to shorten telomere lengths in human cells. ${ }^{19}$ Using qPCR we measured the lengths of telomeres in the treated and untreated cells (Figure 5a). The results show that HEK cells have on average $10.2 \pm 0.12 \mathrm{kbps}$ long telomeres, whereas the HEK-AZT treated cells have on average $9.2 \pm 0.12 \mathrm{kbps}$ long telomeres, which is significantly different in a $t$ test at $P=0.005$. Figure $5 b$ shows the equivalent diameter of $>1500$ super resolution measured telomeres from treated and untreated cells. 

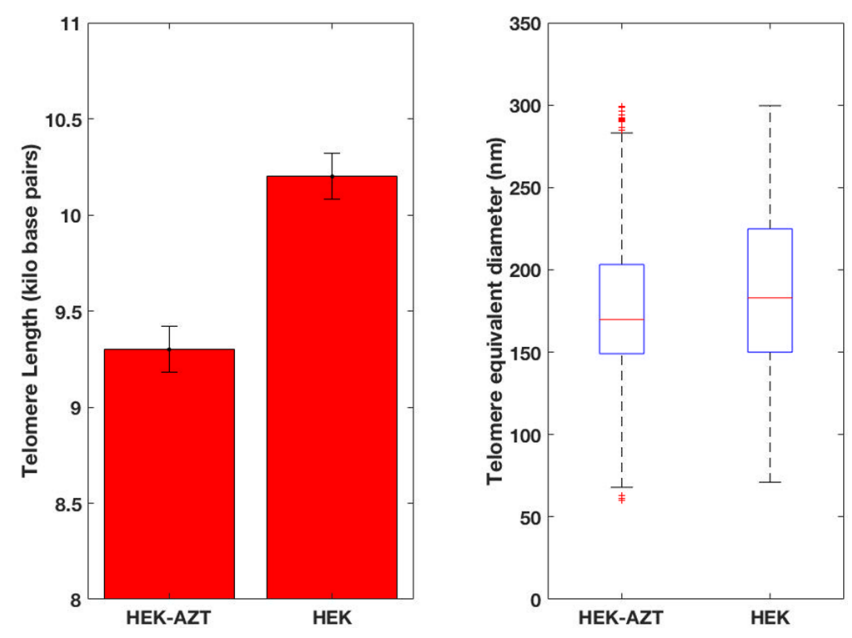

Figure 5. The comparison of telomere properties in control (HEK) and drug-treated (HEK-AZT) cells. (a) The absolute length of telomeres measured by quantitative polymerase chain reaction (qPCR). The error bars show the standard deviation. The drugtreated cells have on average $\sim 1 \mathrm{kbp}$ shorter telomeres. (b) The diameters of telomeres $(N>1500)$ in control vs drug-treated were measured using super resolution microscopy. The ranges were between $\sim 60$ and $300 \mathrm{~nm}$, but the drug treated cells had a slightly lower median $(169 \mathrm{~nm})$ than the control $(183 \mathrm{~nm})$, which was significant different in a Mann-Whitney- $U$ test at $P=0.005$.

Here, the median diameter of the untreated HEK and AZT treated cells is 183 and $169 \mathrm{~nm}$, respectively, which is significantly different at $P=0.005$ using a Mann-Whitney-U test. Clearly the ranges overlap considerably with a spread of diameters from $\sim 60-300 \mathrm{~nm}$. Although the difference is small, it is still just detectable and mirrors the results seen with the absolute telomere values measured with qPCR.

\section{DISCUSSION}

We have developed a gold nanoparticle probe, designed to measure length and absolute dimensions of telomeres and have evaluated its specificity and sensitivity. Over the past 20 years, considerable efforts have been made to accurately measure the length, biophysical volume, and density of telomeres, due to their importance in many areas of aging and cell cycle regulation. Here, we will discuss the sensitivity and specificity of our GNP probe and then consider the implications for our super resolution results on the diameters of telomeres.

We chose synchrotron X-ray fluorescence to measure the length of telomeres, as it has the potential to absolutely quantify gold with micron resolution, and a beam energy which can be "tuned" to optimize signal from an element of interest, giving it very high sensitivity ( $\mathrm{ppm})$. To our knowledge, no attempt has been made to measure telomeres with an XRF-compatible probe, although similar probes have been made for Raman spectroscopy. ${ }^{19}$ Indeed, our group has used ion beam analysis (a closely related XRF technique) to absolutely quantify the numbers of gold nanoparticles and titania nanoparticles in individual cells as well as other endogenous biological trace elements such as $\mathrm{Ca}, \mathrm{P}, \mathrm{Na}, \mathrm{K}, \mathrm{S}^{20-22}$

Analysis of our XRF data indicate that there is on average $\sim 0.5$ pg of gold in probed cell nuclei or about 4400 GNPs. This corresponds to binding $\sim 920$ bases of DNA if we assume that each GNP binds to 18 bases and each nucleus has 86 telomeres. Using $\mathrm{qPCR}$ which is a conventional method to measure telomere length, we showed that our HEK cells had telomeres $\sim 10 \mathrm{kbps}$ long. This means that the sensitivity of our probe is $\sim 10 \%$.

The reason for the low sensitivity is probably due to steric interference that the $10 \mathrm{~nm}$ GNPs experience with the chromosome structure. That said, we initially chose $10 \mathrm{~nm}$ GNPs as previous reports from Zong et al. ${ }^{19}$ had shown good Raman signals from similarly designed $20 \mathrm{~nm}$ gold nanoparticle telomere probes. Zong et al. did not do a sensitivity analysis of their probe, rather they compared Raman signal to a centromeric silver probe and measured ratios of gold to silver signal as a means to compare telomere length on drug treated vs untreated cells. Our results indicate that is it very unlikely that their $20 \mathrm{~nm}$ sized probe had full coverage of the telomere length.

As a way to improve sensitivity, ultrasmall NPs $(\sim 1.5 \mathrm{~nm})$ could be used instead of $10 \mathrm{~nm}$ NPs. The caveat here is that there will be less gold atoms to detect per probe, perhaps beyond the sensitivity of XRF. On the other hand, the coverage with ultrasmall gold nanoparticles will likely be much greater, and so there may actually be similar numbers of gold atoms than with larger, but fewer, gold nanoparticles. Indeed, XRF does have the sensitivity to measure $2 \mathrm{~nm}$ gold nanoparticles linked to antibodies in ppm concentrations. ${ }^{23}$

We then measured the specificity of our probe to be $~ 90 \%$, comparable to a standard QFISH probe. In fact, it is not surprising that the specificity of our probe is good, as the PNA part is identical to a conventional QFISH probe, which has proven excellent specificity. ${ }^{10}$ However, there is always the possibility of nonspecific interactions between DNA and gold nanoparticles. However, we believe the stringent washes $\left(65^{\circ} \mathrm{C}\right.$ in $0.2 \%$ detergent) we performed after hybridization (see the FISH protocol) satisfactorily removed almost all nonspecifically bound probe.

We then used the Alexa647 part of the probe to measure telomeres using super resolution microscopy, which unlike diffraction-limited microscopy gives realistic dimensions. The following discussion describes how even with very large differences in telomere lengths, super resolution actually has relatively poor sensitivity in measuring these differences as a volume or diameter. This means that caution should be used interpreting results measuring compact vs decompact or long vs short telomere regions.

There are very few studies to date where super resolution techniques have been applied to telomeres. ${ }^{4,5,24}$ However, currently there is particular interest in measuring telomere biophysical properties, as there is controversy about whether DNA compaction influences the molecular interaction between enzyme complexes and telomeric DNA, and in particular, how to interpret the effect of shelterin.

Bandaria et al. $^{4}$ recently measured the volumes of telomeres by super resolution microscopy in compact vs decompact telomeres, using various shelterin complex knockout cell lines. They proposed that shelterin protects chromosome ends by compacting the telomeric chromatin, thus preventing DNA damage response enzymes from entering into the telomeric space. More recently, the opposite effect was found by Timashev et al. ${ }^{5}$ and Vancevska et al. ${ }^{6}$ who found that telomeric DNA damage response occurs in the absence of DNA decompaction and questioned whether shelterin really compacted DNA to any substantial degree. An important issue in this debate is measuring the packing density of the telomeres, which our results allow, as we have measured both 
the realistic length (using qPCR) and the actual diameter (with super resolution).

First, it is important to summarize telomere diameter results from the literature values and compare them to our values. We found that HEK cells had an average telomere length of $\sim 10$ kbps, while they had an average diameter of $\sim 180 \mathrm{~nm}$ and a range between 60 and $300 \mathrm{~nm}$. This in good agreement with literature values that range between $\sim 60$ and $400 \mathrm{~nm}$, depending on the cell line and conditions used. In particular, Vancevska et al. showed that HeLa cells with "long" telomeres (average $\sim 30 \mathrm{kbps}$ ) had an average diameter of $\sim 190 \mathrm{~nm}$ (range 100-300 nm) while HeLa cells with "short" telomeres had an average diameter of $\sim 130 \mathrm{~nm}$ (range 60-200 nm). Timashev et al., using the mouse MEF cell line, reported slightly larger values, ranging from $\sim 80$ to $400 \mathrm{~nm}$ with a mean diameter $\sim 200 \mathrm{~nm}$. (Note: both these papers report radius rather than diameter). Bandaria et al. used HeLa cells and report volumes of telomeres, giving an average diameter of $\sim 150 \mathrm{~nm}$. Overall, there is very good agreement with the reported values and our own.

From our telomere diameter measurements, we can make an estimation of the DNA spacing or compaction. If we consider DNA as a cylinder, the volume is defined by the length and radius of a base pair ( $0.332 \mathrm{~nm}$ length, $1 \mathrm{~nm}$ radius), so that the volume of $10 \mathrm{kbps}$ is theoretically $1.25 \times 10^{4} \mathrm{~nm}^{3}$. The average HEK telomere volume is actually $\sim 140$ times more than this (sphere with diameter $180 \mathrm{~nm}$ or $1.76 \times 10^{6} \mathrm{~nm}^{3}$ ). For a point of reference, the "average" packing density or spacing of genomic DNA is $\sim 120$ times more than the theoretical limit (genomic DNA $=\sim 3.2$ billion base pairs or $3.33 \times 10^{9} \mathrm{~nm}^{3}$; volume of a spherical $5.5 \mu \mathrm{m}$ radius cell nucleus $=2.94 \times 10^{11}$ $\mathrm{nm}^{3}$ ). So we can estimate that the telomeric DNA is similarly spaced in comparison with "average" genomic DNA. This is interesting as it is often assumed that telomeres are much more compact than "average" DNA, but as we see in the following discussion, even when proteins are deleted that were thought to have a "compaction" effect, there is hardly a change in the measured diameters of telomeres.

We also treated HEK cells with a purported telomerase inhibitor, AZT. We did this to have a direct comparison between "long" vs "short" telomeres. With qPCR, we measured a significantly reduced average telomere length of drug treated HEK cells (about $1 \mathrm{kbps}$ : $\sim 9 \mathrm{kbps}$ drug-treated compared to $\sim 10 \mathrm{kbps}$ in controls). This difference could just be seen using super resolution microscopy, where we measured diameters of individual telomeres with $\sim 170 \mathrm{~nm}$ drug-treated vs $\sim 180 \mathrm{~nm}$ control, although the ranges of the two samples overlapped considerably.

Similarly, Vancevska et al. compared the sensitivity of super resolution microscopy in measuring the difference between "long" vs "short" telomeres, against a conventional method (Southern hybridization). Here, they used two different strains of HeLa cells genetically modified for cells with long or short telomeres. With southern hybridization there was a clear telomere length difference, where an average "long" cell had telomeres that were $\sim 30 \mathrm{kbps}$, while the average "short" cell had $\sim 10$ kbps. With super resolution, the "short" cells had a mean telomere radius of $\sim 68 \mathrm{~nm}$ while the "long" cells had a $\sim 90 \mathrm{~nm}$, with large overlapping ranges between the two. So, with a conventional technique there was a clear difference, while with super resolution it was measurable, but much less pronounced. Similarly we found that a clear difference in qPCR manifests as only a very marginal difference with super resolution microscopy (see Figure 5).

The point is that measuring compact vs decompact, or long vs short telomere regions, with super resolution is challenging due to the large range of telomere diameter values, and results should be interpreted with caution.

The real strength of super resolution lies in the ability of analyzing individual telomeres particularly when colocalizing proteins and complexes within the telomeric space, or visualizing the telomere DNA loop as Doksani et al. ${ }^{24}$ achieved.

\section{CONCLUSIONS}

We have developed and evaluated a dual purpose probe for human telomeres. We measured the gold signal arising from this probe located on telomeres inside cell nuclei. We measured approximately 4400 GNPs per nucleus. This gold content indicates a probe sensitivity of $\sim 10 \%$, based on comparisons with $\mathrm{qPCR}$ based telomere length measurements. The low sensitivity is probably due to steric interference of a relatively large GNP size not gaining full access to the telomeric space. We then measured the probe specificity to be $\sim 90 \%$, by comparing silver enhanced samples (which directly indicate the locations of gold nanoparticles) to a standard QFISH probe. We then used the fluorescent part of our GNP-PNA-A647 probe to measure realistic diameters of human telomeres. Here, we show that the average diameter is about $180 \mathrm{~nm}$ and that the telomeres are rarely circular. This is important as it relates to the packing density of the DNA and hence how accessible telomeres are to various modifying enzymes. Further, by comparing telomeres from drug treated cells that have on average $1 \mathrm{kbps}$ less DNA in the telomeres, we are able to discuss the sensitivity of super resolution as a technique compared to conventional methods.

This work has laid the foundation for a probe design that will be able to simultaneously measure the absolute length and biophysical dimensions of individual telomeres in human cells. Moreover, the approach could have wider uses in measuring other important biomolecules inside cells and tissue. The absolute size of structures and the number of molecules present are often important parameters for understanding basic biological processes.

\section{EXPERIMENTAL METHODS}

Gold Nanoparticle Probe Construct. Briefly, the GNPs are $\sim 10$ $\mathrm{nm}$ in size, coated with polyethylene glycol (PEG) terminated with $\mathrm{NH}$ moieties (see the manufacturer's Web site for physical characteristics). The probe is an antisense 18-mer peptide nucleic acid corresponding to the telomeric repeat on the chromosomes of (GGGTAA), with a spacer molecule and lysine residue at both ends, and an Alexa-647 molecule at the NH terminus end (we call it PNAA647). The GNPs are mixed with the PNA-A647 in a molar ratio of $1: 100$, respectively, left to react for $2 \mathrm{~h}$, centrifuged at $17000 \mathrm{~g}$ and washed three times to remove any unbound PNA-A647 (details can be found in the Supporting Information). Absorbance spectroscopy of the GNP-PNA-A647 conjugate shows two clear peaks at $\sim 520 \mathrm{~nm}$ and $\sim 655 \mathrm{~nm}$ associated with absorption from the GNPs and the Alexa647 dye, respectively, indicating the PNA-A674 has bound to the GNPs (see the Supporting Information).

Cell Culture. The HEK-293 cells were a gift from Dr. Mark Russell (University of Exeter). The cells originated from ATCC cell culture bank and were grown according to standard protocols. They were incubated at $37^{\circ} \mathrm{C}$ in EMEM media with $10 \%$ FBS and passaged every 2-3 days. We avoided the use of antibiotics.

Metaphase Spread Protocol. Cells were grown to $\sim 70 \%$ confluence in T75 (Thermo Fisher Scientific, U.K.) flasks, yielding 
$\sim 1 \times 10^{7}$ cells. Next, the metaphase-blocking drug KaryoMax colcemid solution (Thermo Fisher Scientific, U.K.) was added at a final concentration of $0.1 \mu \mathrm{g} / \mathrm{mL}$ and incubated with the cells for $4 \mathrm{~h}$. The cells were harvested by incubating with trypsin and then centrifuged at $300 \mathrm{~g}$ for $5 \mathrm{~min}$ to pellet the cells. After aspirating the trypsin, $5 \mathrm{~mL}$ of $0.068 \mathrm{M} \mathrm{KCl}$ was added in drops to resuspend the pellet, mixing gently with each drop. After leaving the solution at room temperature for $15 \mathrm{~min}, 0.5 \mathrm{~mL}$ of ice-cold fixative (three parts absolute methanol to one part glacial acetic acid) was added. After a further centrifugation step and aspiration of the supernatant, the cells were resuspended in $5 \mathrm{~mL}$ of the fixative and stored at $-20{ }^{\circ} \mathrm{C}$.

We used two different substrates to drop the cells onto; we used a silcon nitride ( $\mathrm{SiN}$ ) window (Silson Ltd., U.K.) for cell nuclei intended to be analyzed by XRF, while we used glass coverslip slides for those analyzed by dSTORM. Silicon nitride is a good substrate for XRF as it contains no trace element impurities. However, $\mathrm{SiN}$ windows are expensive and fragile so processing cells on them with multiple washing and viewing is technically difficult as they have a tendency to break.

To obtain good quality metaphase spreads, cells were "dropped" from a pipet (each drop contains about $20 \mu \mathrm{L}$ ) from a height of about $2 \mathrm{~cm}$, onto a substrate ( $\mathrm{SiN}$ window or coverslip slide) placed at a 45degree angle. We did this over a water bath to maintain a high humidity environment to prevent the chromosomes from drying too quickly.

Fluorescent in Situ Hybridization Protocol. We processed the metaphase spreads we had prepared on either coverslip slides or $\mathrm{SiN}$ windows using the DAKO (DAKO Ltd., Denmark) FISH telomere kit (code K5236). This kit provides all key reagents needed for performing fluorescence in situ hybridization for detection of telomere sequences by fluorescence microscopy. The metaphase spreads are first fixed in 4\% paraformaldehyde. After 2 washes with Tris Buffered Saline (TBS), $\mathrm{pH} 7.5$, a pretreatment solution of proteolytic enzyme (proteinase $\mathrm{K}$ ) is left on the samples for $10 \mathrm{~min}$ to remove all the proteins. After further washes in TBS and dehydration in ethanol, 10 $\mu \mathrm{L}$ of the GNP-PNA-A647 probe (stock solution diluted 1:100) was added to the sample. The samples were then placed in an oven at 80 ${ }^{\circ} \mathrm{C}$ for 5 min under a coverslip, to denature the DNA in the presence of the probe. Next the samples were placed in the dark at room temperature (RT) for $30 \mathrm{~min}$ to allow hybridization to take place.

The hybridization was followed by a brief rinse with a Rinse Solution (proprietary solution, DAKO kit), and a posthybridization wash with a Wash Solution ( $0.1 \%$ triton-X in TBS) at $65^{\circ} \mathrm{C}$ for $5 \mathrm{~min}$. Following this, the samples were dehydrated in ethanol and left to airdry.

Assessment of Telomere Length by qPCR. DNA was extracted from HEK-293 cells using the PureLink Genomic DNA Mini Kit (Invitrogen/Thermo Fisher, MA) according to the manufacturer's instructions. DNA quality and concentration was checked by Nanodrop spectrophotometry (NanoDrop/Thermo Fisher, MA). Telomere length was determined using a modified qPCR protocol. ${ }^{17}$ PCR reactions contained $1 \mu \mathrm{L}$ of EvaGreen (Solis Biodyne, Tartu, Estonia), $2 \mu \mathrm{M}$ each primer, and $25 \mathrm{ng}$ of DNA in a total volume of 5 $\mu \mathrm{L}$. The quantitative real time polymerase chain reaction telomere assay was run on the StepOne Plus, cycling conditions were a single cycle of $95{ }^{\circ} \mathrm{C}$ for $15 \mathrm{~min}$ followed by 45 cycles of $95{ }^{\circ} \mathrm{C}$ for $10 \mathrm{~s}, 60$ ${ }^{\circ} \mathrm{C}$ for $30 \mathrm{~s}$, and $72{ }^{\circ} \mathrm{C}$ for $1 \mathrm{~min}$. A standard curve is established by dilution of known quantities of a synthesized 84 oligonucleotide containing only TTAGGG repeats. Using the standard curve method, cycle threshold values were plotted on the standard curve to estimate a concentration value for telomere DNA repeat sequences. The average telomere length was calculated as the ratio of telomere repeat copy number to a single gene (36B4) copy number.

X-ray Fluorescence. The Diamond Light source beamline uses a pair of KirkPatrick-Baez focusing mirrors to deliver a tunable size beam to the sample, in our case it was approximately $2.5 \times 2.5 \mu \mathrm{m}^{2}$. A silicon drift detector was used at $45^{\circ}$ geometry to collect the characteristic photons from the elements in the sample. The excitation energy was set at $12.5 \mathrm{keV}$.
Super Resolution Microscope. The super resolution images were taken using a custom-made microscope. The methodology used to take images and process the data have been published previously. ${ }^{18}$ The coverslips were fixed to a custom-made chamber such that the nuclei could be covered with an oxygen-scavenging solution $(0.03 \mathrm{M}$ MEA [mercaptoethylamine]) in glycerol buffered with $1 \times$ PBS (phosphate buffered saline), optimized for A647 photoswitching. Images were acquired on a commercial Nikon Ti-E inverted microscope with a Nikon $60 \times, 1.49$ NA oil-immersion TIRF objective (Nikon), and an Andor Zyla 4.2 sCMOS camera (Andor Technology). Cell nuclei were found using the DAPI signal from stained chromosomes using $405 \mathrm{~nm}$ illumination from an LED light source. The A647 dye bound to the telomeres on the ends of the chromosomes was illuminated with a $645 \mathrm{~nm}$ LED, combined with a Cy5 filter cube (Semrock, 655/40). A region of interest (ROI) was imaged first with LED illumination and then in super resolution mode. For dSTORM, illumination was by a $642 \mathrm{~nm}$ laser (Omicron, LuxX $642-140)$ providing approximately $100 \mathrm{~W} / \mathrm{m}^{2}$ focal plane intensity with a spot size diameter of about $40 \mu \mathrm{m}$. Cells were illuminated at high intensity for $\sim 5 \mathrm{~s}$ to push a large proportion of the dye molecules into a dark state, whereupon frames were recorded at $50 \mathrm{~ms}$ intervals with custom software detecting single molecule events in real time. We collected approximately 20000 events per ROI.

Images were reconstructed using custom software, written in Python. ${ }^{18}$ Briefly, each event was processed using a "jittered triangulation" algorithm, which essentially produces a 64 bit matrix (and thus an image), where the value of each event is weighted in comparison to the closeness of its neighbor.

Image Analysis. Each super resolution image was saved as a TIF file and was further processed in MATLAB. The image analysis code and example images can be found on github.com/charliejeynes. Briefly, each image is binarized using an Otsu threshold. Then the binarized LED image is compared to the binarized super resolution image, so that only dots that register in the same place in both images (i.e., are truly telomeres and not background dots) are counted. Next, each telomere region is then measured with a number of parameters including area, perimeter, and equivalent diameter. Throughout the paper we use equivalent diameter as it normalizes the often-irregular shapes of the telomeres. The equivalent diameter is calculated as the $\sqrt{ }(4 \times$ area $/ \pi)$.

Merged LED and super resolution images shown in Figure 3 were made from the DAPI (blue) channel for the chromosomes and the A647 (red channel) for the telomeres. Image analysis of the gold nanoparticle organic shells shown in Figure 1 followed a very similar image analysis pipeline to that described above for the super resolution images.

\section{ASSOCIATED CONTENT}

\section{S Supporting Information}

The Supporting Information is available free of charge on the ACS Publications website at DOI: 10.1021/acsnano.7b07064.

Conjugation details for the gold nanoparticle probe, thickness measurements for the cell nuclei using RBS and STIM, and additional measurements of nuclei with XRF (PDF)

\section{AUTHOR INFORMATION}

\section{Corresponding Author}

*E-mail: c.jeynes@exeter.ac.uk.

\section{ORCID}

J. Charles G. Jeynes: 0000-0002-4354-3852

Andrew A. Bettiol: 0000-0001-5242-3644

\section{Notes}

The authors declare no competing financial interest.

The image analysis code and example images can be found on github.com/charliejeynes. 


\section{ACKNOWLEDGMENTS}

J.C.G.J. thanks Vladimir Palitsin for work setting up the Surrey Ion Beam Centre microbeam, Ruisheng Lin for valuable help on the super resolution system, and Prof. Frank Watt for valuable discussion. This work was generously supported by the Wellcome Trust Institutional Strategic Support Award (Grant WT105618MA).

\section{REFERENCES}

(1) Aubert, G.; Lansdorp, P. M. Telomeres and Aging. Physiol. Rev. 2008, 88, 557-579.

(2) de Lange, T. How Telomeres Solve the End-Protection Problem. Science 2009, 326, 948-952.

(3) Soudet, J.; Jolivet, P.; Teixeira, M. T. Elucidation of the DNA End-Replication Problem in Saccharomyces cerevisiae. Mol. Cell 2014, 53, 954-964.

(4) Bandaria, J. N.; Qin, P. W.; Berk, V.; Chu, S.; Yildiz, A. Shelterin Protects Chromosome Ends by Compacting Telomeric Chromatin. Cell 2016, 164, 735-746.

(5) Timashev, L. A.; Babcock, H.; Zhuang, X. W.; de Lange, T. The DDR at Telomeres Lacking Intact Shelterin Does not Require Substantial Chromatin Decompaction. Genes Dev. 2017, 31, 578-589.

(6) Vancevska, A.; Douglass, K. M.; Pfeiffer, V.; Manley, S.; Lingner, J. The Telomeric DNA Damage Response Occurs in the Absence of Chromatin Decompaction. Genes Dev. 2017, 31, 567-577.

(7) Aubert, G.; Hills, M.; Lansdorp, P. M. Telomere Length Measurement-Caveats and a Critical Assessment of the Available Technologies and Tools. Mutat. Res., Fundam. Mol. Mech. Mutagen. 2012, 730, 59-67.

(8) Bekaert, S.; Koll, S.; Thas, O.; Van Oostveldt, P. Comparing Telomere Length of Sister Chromatids in Human Lymphocytes Using Three-Dimensional Confocal Microscopy. Cytometry 2002, 48, 34-44.

(9) Lansdorp, P. M.; Verwoerd, N. P.; vandeRijke, F. M.; Dragowska, V.; Little, M. T.; Dirks, R. W.; Raap, A. L.; Tanke, H. J. Heterogeneity in Telomere Length of Human Chromosomes. Hum. Mol. Genet. 1996, $5,685-691$.

(10) Poon, S. S. S.; Martens, U. M.; Ward, R. K.; Lansdorp, P. M. Telomere Length Measurements using digital fluorescence microscopy. Cytometry 1999, 36, 267-278.

(11) Deng, W.; Tsao, S. W.; Lucas, J. N.; Leung, C. S.; Cheung, A. L. $\mathrm{M}$. A new method for Improving Metaphase Chromosome Spreading. Cytometry 2003, 51A, 46-51.

(12) van de Linde, S.; Kasper, R.; Heilemann, M.; Sauer, M. Photoswitching Microscopy with Standard Fluorophores. Appl. Phys. B: Lasers Opt. 2008, 93, 725-731.

(13) del Pino, P.; Yang, F.; Pelaz, B.; Zhang, Q.; Kantner, K.; Hartmann, R.; de Baroja, N. M.; Gallego, M.; M?ller, M.; Manshian, B. B.; Soenen, S. J.; Riedel, R.; Hampp, N.; Parak, W. J. Basic Physicochemical Properties of Polyethylene Glycol Coated Gold Nanoparticles that Determine Their Interaction with Cells. Angew. Chem., Int. Ed. 2016, 55, 5483-5487.

(14) Mosselmans, J. F. W.; Quinn, P. D.; Rosell, J. R.; Atkinson, K. D.; Dent, A. J.; Cavill, S. I.; Hodson, M. E.; Kirk, C. A.; Schofield, P. F. The First Environmental Science Experiments on the New Microfocus Spectroscopy Beamline at Diamond. Mineral. Mag. 2008, 72, 197200.

(15) Jeynes, C.; Barradas, N. P.; Szilagyi, E. Accurate Determination of Quantity of Material in Thin Films by Rutherford Backscattering Spectrometry. Anal. Chem. 2012, 84, 6061-6069.

(16) Chen, X.; Chen, C. B.; Udalagama, C. N. B.; Ren, M. Q.; Fong, K. E.; Yung, L. Y. L.; Giorgia, P.; Bettiol, A. A.; Watt, F. HighResolution 3D Imaging and Quantification of Gold Nanoparticles in a Whole Cell Using Scanning Transmission Ion Microscopy. Biophys. J. 2013, 104, 1419-1425.

(17) O'Callaghan, N. J.; Dhillon, V. S.; Thomas, P.; Fenech, M. A Quantitative Real-Time PCR Method for Absolute Telomere Length. BioTechniques 2008, 44, 807-809.
(18) Baddeley, D.; Jayasinghe, I. D.; Lam, L.; Rossberger, S.; Cannell, M. B.; Soeller, C. Optical Single-Channel Resolution Imaging of the Ryanodine Receptor Distribution in Rat Cardiac Myocytes. Proc. Natl. Acad. Sci. U. S. A. 2009, 106, 22275-22280.

(19) Zong, S. F.; Chen, C.; Wang, Z. Y.; Zhang, Y. Z.; Cui, Y. P. Surface Enhanced Raman Scattering Based in situ Hybridization Strategy for Telomere Length Assessment. ACS Nano 2016, 10, $2950-2959$.

(20) Jeynes, J. C. G.; Bailey, M. J.; Coley, H.; Kirkby, K. J.; Jeynes, C. Microbeam PIXE Analysis of Platinum Resistant and Sensitive Ovarian Cancer Cells. Nucl. Instrum. Methods Phys. Res., Sect. B 2010, 268, $2168-2171$

(21) Jeynes, J. C. G.; Jeynes, C.; Merchant, M. J.; Kirkby, K. J. Measuring and Modelling Cell-to-Cell Variation in Uptake of Gold Nanoparticles. Analyst 2013, 138, 7070-7074.

(22) Jeynes, J. C. G.; Jeynes, C.; Palitsin, V.; Townley, H. E. Direct Quantification of Rare Earth Doped Titania Nanoparticles in Individual Human Cells. Nanotechnology 2016, 27, 285103.

(23) McRae, R.; Lai, B.; Vogt, S.; Fahrni, C. J. Correlative MicroXRF and Optical Immunofluorescence Microscopy of Adherent Cells Labeled with Ultrasmall Gold Particles. J. Struct. Biol. 2006, 155, 2229.

(24) Doksani, Y.; Wu, J. Y.; de Lange, T.; Zhuang, X. W. SuperResolution Fluorescence Imaging of Telomeres Reveals TRF2Dependent T-loop Formation. Cell 2013, 155, 345-356. 